\title{
The design of molecular switches for biosensors
}

\begin{abstract}
A biosensor is a device, which incorporates a biological sensing element near to or integrated with a physical signal transducer. The sensing elements accomplish recognition from the binding, which occurs between bio recognition molecules, and target analytes. Transduction is the physicochemical perturbation caused by this binding that enables recognition of the triggered change by some device. To detect a small number of binding events, a single binding event must be amplified. Here, we design a molecular switch capable of converting a single binding event into the movement of about one million ions per second. In this switch, a single binding, amplified by the release of the stored free electrochemical energy, leads to a dynamic signal that is large compared to the noise in the measuring system. This approach is adapted from biological receptors, which convert chemical signals into currents in ion channels. The molecular switches in this work are artificial ion channels constructed by modular design from molecular pores and gates. The currents through these channels can be registered by conventional methods. The molecular switches can be triggered by various sensing elements such as antibodies, antibody fragments, polypeptides, DNA, RNA, and ion sensitive molecules. The small size and planar architecture of the molecular switches allow them to become components of a microelectronic circuit. The switches can be used for detection of proteins, toxins, viruses, bacteria, and ions.
\end{abstract}

Keywords: signal transduction, modular design, ion channel, monolayer, receptor, antibody, membrane
Volume 2 Issue I - 2017

\author{
Vitaly Vodyanoy \\ Department of Anatomy, Physiology and Pharmacology, Auburn \\ University, USA
}

Correspondence: Vitaly Vodyanoy, Department of Anatomy, Physiology and Pharmacology, 207 Greene Hall, Auburn University, Auburn, AL 36849, USA, Telephone 334-844-5405, Fax 334-844-5388,Email vodyavi@auburn.edu

Received: December 04, 2016 | Published: January 23, 2017

\section{Introduction}

In nature, specialized proteins, specific receptors on or in target cells, recognize signaling molecules. ${ }^{1}$ They specifically bind the signaling molecule and then initiate a response in the target cell. Many receptors use the transduction mechanism utilizing ion channels. These so-called, ion-channel-linked receptors, are also known as transmitter-gated ion channels. The signaling is mediated by binding of a specific molecule, which that opens or closes the gate of the ion channel, thereby changing the ion permeability and electrical conductance of the channel.

Thus, these transmitter-gated ion channels work as chemo electric transducers. There are several molecular mechanisms of opening and closing of natural and artificial ion channels. ${ }^{2}$ Channels can be opened or closed as a result of aggregation - disaggregation of molecular subunits constituting a channel (Figures 1A) (Figure 1B) or, a molecular gate can control the closing and opening of the channel (Figure 1C). A model system with an antibiotic, gramicidin, where two half-channels connect up, was used to construct a biosensor. ${ }^{3}$ The "off" state of the channel was realized by the displacement of one-half of the channel due to binding of the analyte.

The chief drawbacks of the gramicidin switch were the need for the displacement of the relatively large gramicidin molecule for switching and the absence of independent gate. Genetically engineered protein pores $^{4}$ provide another approach to making molecular switches for biosensors. Protein pores could provide speed and sensitivity as transducers. However, these relatively large protein molecules are difficult to maintain outside the living cell.
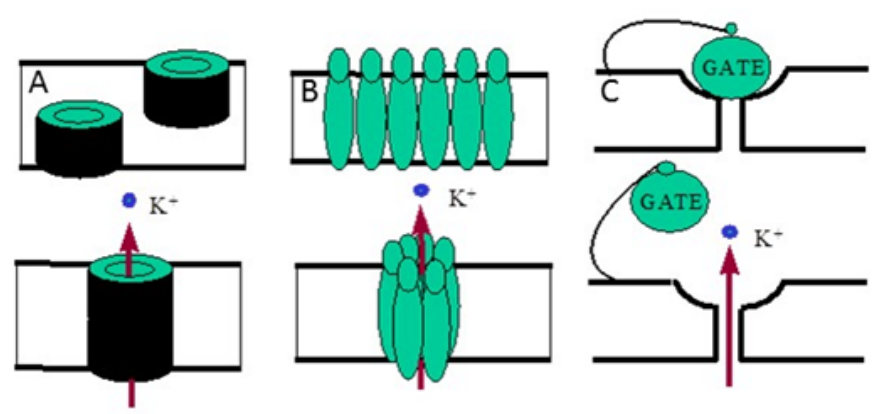

Figure I Mechanisms of opening and closing of ion channels. A, B Aggregation - disaggregation of molecular subunits of a channel. C. Molecular gate.

\section{Materials and methods}

\section{Modular design}

The task of this article is the design of a molecular switch capable of amplification and transduction of a chemical signal into an electrical current to be registered by a conventional electronic instrument. Pore-forming polyene antibiotics and synthetic channel gates immobilized in the artificial supported planar membrane are to be used to compose a modular molecular system capable of switching ion channels mediated by specific binding. Two molecular models are considered for practical applications in biosensors. One molecular switch is intended to be in the NORMALLY "ON" state, which can go to the "OFF" state when a specific molecule is bound to a trigger site. Another switch has a NORMALLY "OFF" state that goes "ON" after a specific binding. In the "ON" state the channel is open and ions 
can go through, while in the "OFF" state the channel is shut off and ions are stopped.

\section{Requirements}

Design an artificial molecular switch capable of performing amplified signal transduction between a variety of chemical sensing elements and an electronic device. The switch needs to be sensitive, fast, and suitable for practical biosensors.

\section{Approach}

For the NORMALLY "ON" switch we use molecules of the polyene antibiotic amphotericin $\mathrm{B}(\mathrm{AmB})$ known to form ion channels in artificial lipid membranes. ${ }^{5-9}$ The presence of the cholesterol molecules in the AmB channel is critical for the channel to be open..$^{10}$ The removal of the cholesterol from the channel shuts the channel off. Thus, the switch, in this case, is the modular assembly of the amphotericin pore and the cholesterol gate tethered with the sensing element (antibody, polypeptide, etc.). The channel is switched when the target is recognized, bound to the sensing element, and the displacement of the cholesterol gate shuts the channel off.

The major modular components of the NORMALLY "ON "molecular switch (Figure 3) are phospholipids for the supporting membrane (Figure 1) (Figure 3A), amphotericin B for construction of the ion channel (Figure 2) ( Figure 3A), the cholesterol gate (Figures $3 \mathrm{~A}, 3 \& 4$ ), and the molecular anchor (Figure 3A) ( Figure 4). Phospholipids and amphotericin B are commercially available from Avanty Polar Lipids, Inc. and Sigma Chemical Co. The displaceable cholesterol gate is composed of the cholesterol molecule linked to an antibody (SynPep CA). The molecular anchor is the same antibody linked to the supporting membrane through stearic acid (SynPep, CA) or biotinylated lipid and streptavidin (Molecular Probs Inc, OR). For the proof of concept, the 7-base peptide specific to skeletal muscle protein as an antibody can be used..$^{11,12}$

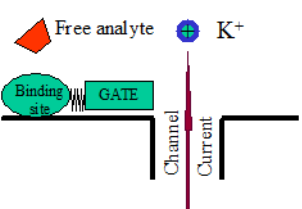

SWITCH IS ON

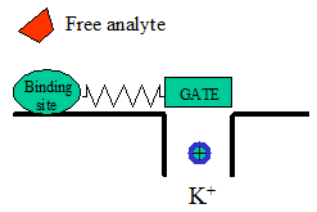

SWITCH IS OFF

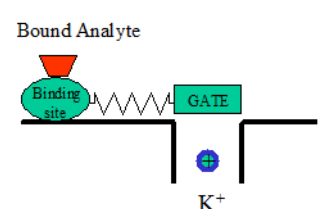

SWITCH IS OFF

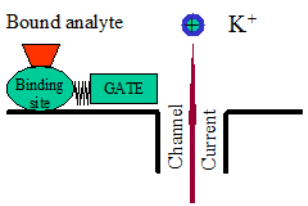

SWITCH IS ON
Figure 2 Normally "ON" and "OFF" switches.

The cholesterol molecule holds the aggregated channel open by zipping the amphotericin molecules together (Figure 3A). When the channel is open, ions can pass through. When the analyte binds the antibody, it displaces the cholesterol and unzips the channel. This results in disaggregation and closing of the channel (Figure 3B). The process is reversible. When an antibody is unbound, the liberated cholesterol can again zip the channel open. The NORMALLY "OFF" switch is constructed as a modular assembly of a small artificial channel and a synthetic molecular gate tethered to the base of the supporting membrane. The channel opens when the target binds the sensing molecules and pulls the plug out of the channel.

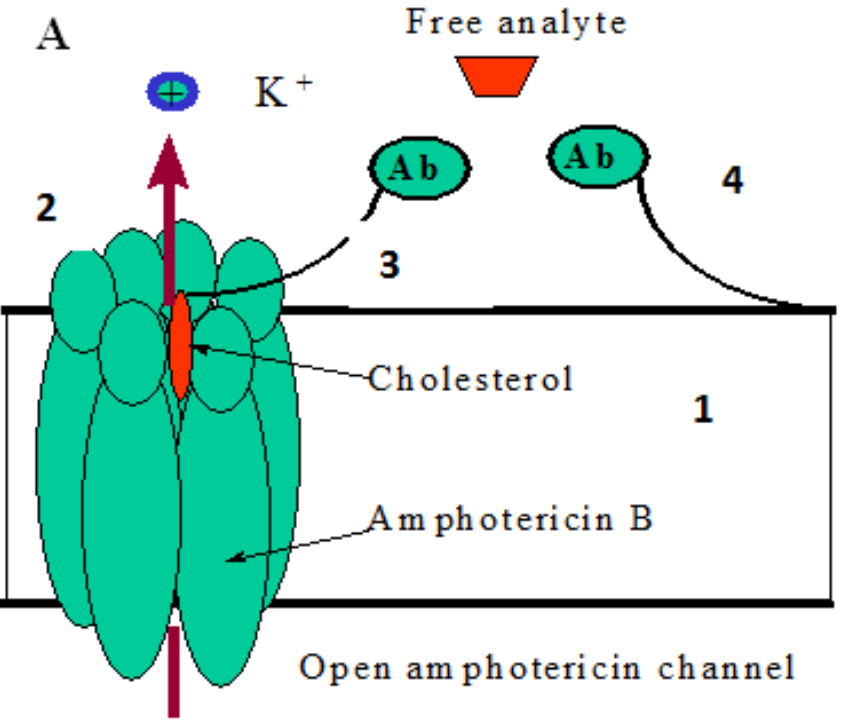

B

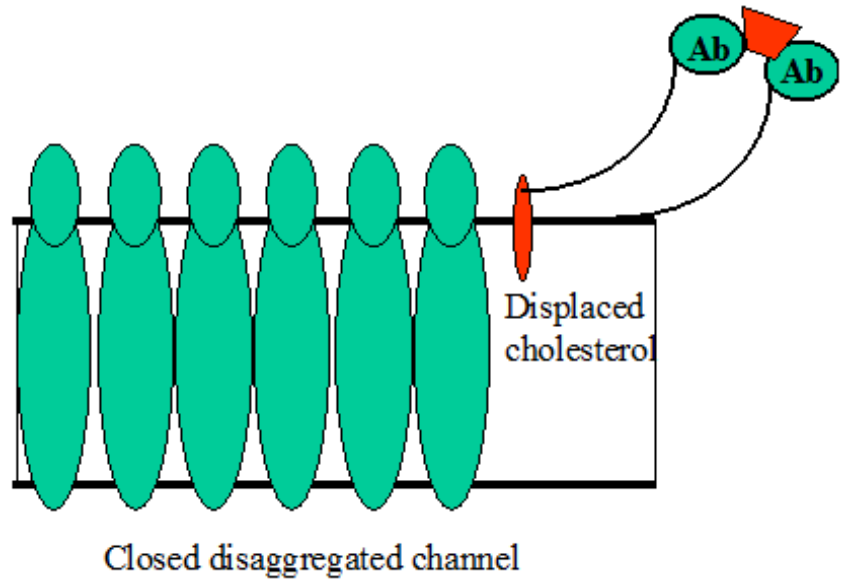

Figure 3 The NORMALLY "ON" switch. The molecular switch assembled from the phospholipid membrane (I), amphotericin B, cholesterol, and antibodies. $\mathrm{K}^{+}$- potassium ion.

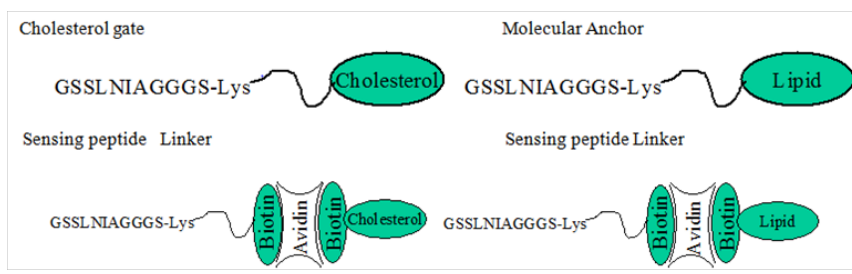

Figure 4 Modular cholesterol gate and molecular anchor.

The major modular components of the NORMALLY "OFF" molecular switch are phospholipids for supporting membrane (Figure 1) (Figure 5A), the amphotericin $B$ and cholesterol for construction of the ion channel (Figure 2) (Figure 5A), the synthetic molecular gate (Figure 3) (Figure 5A), and molecular anchor (Figure 4) (Figure 5A). The supporting membrane, the open amphotericin $\mathrm{B}$-cholesterol channel, and the molecular anchor are the same as in the NORMALLY "ON" switch. The displaceable cholesterol gate is 
unused for this switch. Instead, we use a synthetic molecular gate. We use a tethered blocker of $\mathrm{K}^{+}$-ions (ion plug), attached to a sensing element and membrane with a flexible molecular linker. Components of the molecular gate are shown in Figure 7. The biotinylated lipid (Figure 1) (Figure 6) and the sensing peptide (Figure 2) (Figure 6) are the same as in the NORMALLY "OFF" switch. The biotinylated channel blocker is constructed by using an extender arm maid of ethylene glycol linkages (Figure 3) (Figure 6) and anion, gate (Figure 4) (Figure 6). Extender arms are to be constructed from the spacerlinked biotins available commercially. The gates are to be constructed from known potassium ion blockers ${ }^{2}$ and spacer-link biotinylated compounds (Table 1) (Table 2).
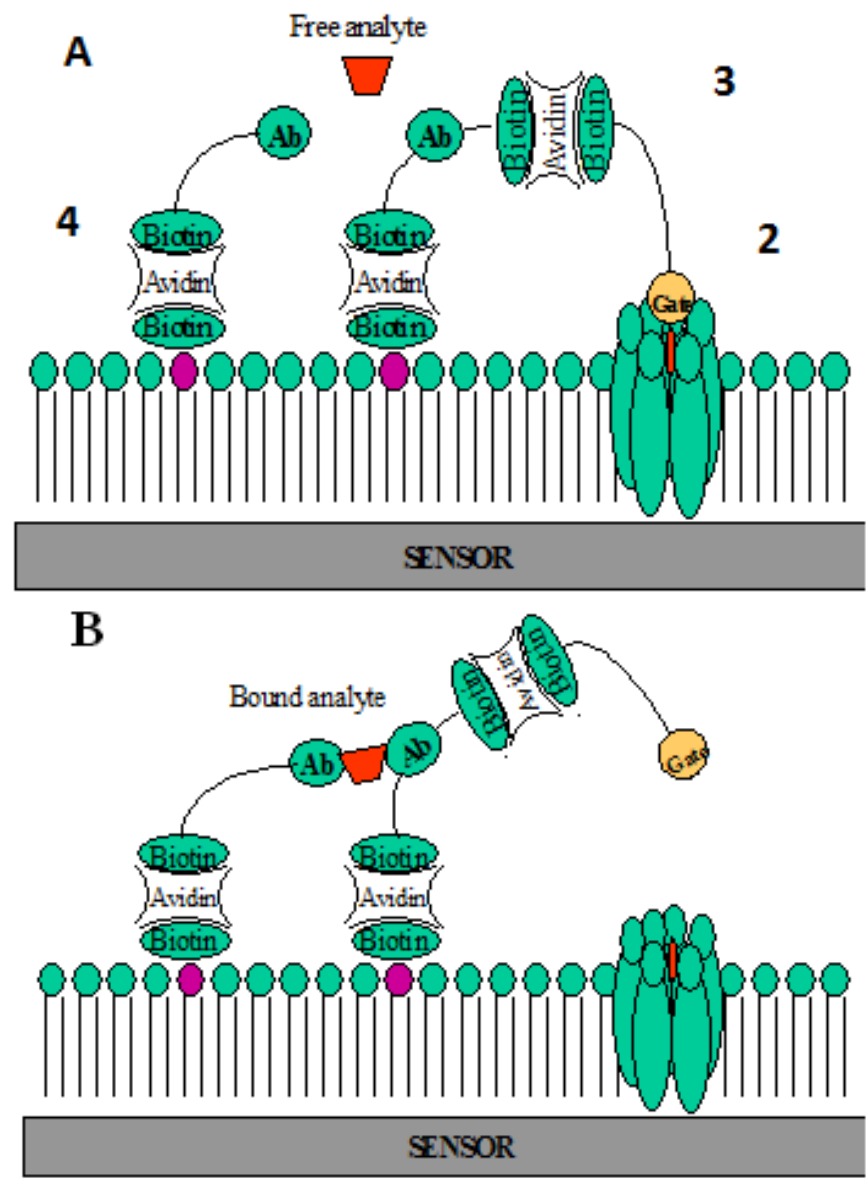

Figure 5 The NORMALLY "OFF" switch. A. Closed channel. The switch is assembled from the phospholipid membrane (I), amphotericin B channel (2), molecular gate (3) and molecular anchor (4). B. Open channel. Analyte binds the sensing peptide and pulls the gate out of the channel.

\section{Immobilization of modular components of the molecular switch}

A critical step in the development of the molecular switch is the immobilization of the modular components, to the platform area on the chip where the target molecules will bind in a positive response. Three methods are used to immobilize molecules on the sensor surfaces ${ }^{1}$ Langmuir-Blodgett (LB) deposition of a composite lipid monolayer onto a sensor surface ${ }^{2,6,13}$ antibody-liposome conjugation ${ }^{14}$ and $^{3}$ molecular self-assembling using biotin/streptavidin coupling. ${ }^{15}$ The precise thickness of LB films, coupled with the degree of control over the molecular architecture, has now firmly established a role for these organic multi layers in thin film technology. Also, LB and molecular assembling technologies can provide a way to produce biocompatible hydrophilic (or hydrophobic) surfaces useful for adsorption of various capture ligands. The LB method uses monolayers containing ligands with high specificity and low non-specific binding. As result, a dynamic equilibrium (association-dissociation) of antibody-antigen interaction can be rapidly achieved and measured. The monolayer in this method is formed on the air-liquid interface by allowing the spreading solution to run down a inclined wettable planar surface that is partially submersed into the sub phase. Membrane vesicles are positioned on the wet slide at the edge of positive meniscus of liquid, at the liquid-air interface. The modular components of the molecular switch are bound to the vesicular membrane. When surface forces rupture the vesicle and it splits into a monolayer the unique process of purification occurs: membrane-bound molecules are left bound to the newly created monolayer, but soluble impurities go into the sub phase beneath the monolayer. When the monolayer is compressed and transferred onto sensor surface there are only membrane-bound molecular components surrounded by compatible lipids. The spreading material is purified in process of spreading, it needs no special preliminary purification. The method is simple and cost-effective.

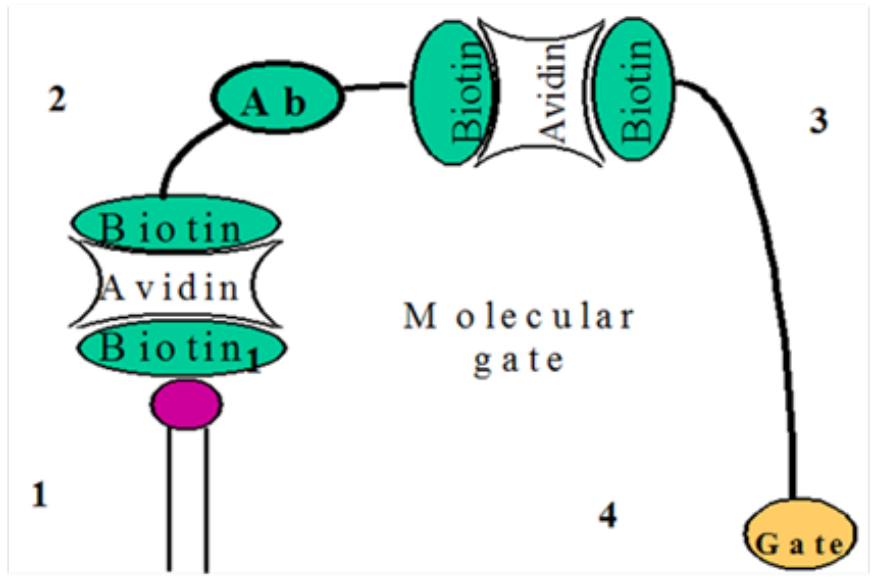

Figure 6 Modular design of the molecular gate: biotinylated lipid (I) sensing peptide (2) linker (3) and ion gate (4).

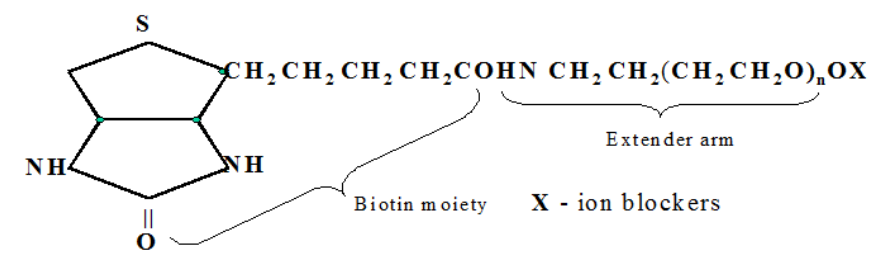

Figure 7 Synthetic molecular gate.

\section{Monolayer formation and deposition for molecular devices}

Phospholipid monolayers: Phospholipid solutions are spread on the surface balance as hexane solutions $(1 \mathrm{mg} / \mathrm{ml})$ containing $2 \%$ ethanol. The sub phase used in the experiments is a solution containing $55 \mathrm{mM} \mathrm{KCl}, 4 \mathrm{mM} \mathrm{NaCl}, 0.1 \mathrm{mM} \mathrm{CaCl}_{2}, 1 \mathrm{mM} \mathrm{MgCl}_{2}$ and 2mM 3-(N-morpholino)-propanesulfonic acid (MOPS) made with de ionized doubly distilled water ( $\mathrm{pH}$ adjusted to 7.4 with $\mathrm{KOH}$ ).

Monolayers made of antibody preparations: The quartz crystals covered with gold are cleaned by treatment with $50 \%(\mathrm{v} / \mathrm{v}) \mathrm{HNO}_{3}$ 
and are rinsed in running distilled water until the acid is completely removed. The crystals are then dried and stored until further use. The monolayer is formed on the air-liquid interface of Lngmuir-Blodget system by allowing the spreading solution to run down a inclined wettable planar surface that is partially submersed into the sub phase. $150 \mu 1$ of the antiserum is spread on the sub phase surface by allowing it to flow down a wet glass plate that crossed the interface. The flow rate down the plate is maintained about $0.1 \mathrm{ml} / \mathrm{min}$. After spreading the glass plate is removed, and the monolayer is allowed to equilibrate and stabilize for 10 minutes at $19 \pm 0.1^{\circ} \mathrm{C}$. The monolayer is then compressed at a rate of $30 \mathrm{~mm} / \mathrm{min}$ and the vertical film deposition is carried out with a vertical rate of $4.5 \mathrm{~mm} / \mathrm{min}$ and at a constant surface pressure of $23 \mathrm{mN} / \mathrm{m}$. Seven monolayers of the antibody (phage, peptide, virus) film are transferred to the gold surface of the quartz crystals in this manner. Monolayers containing antibodies are transferred at a constant surface pressure onto a silicon/silicon dioxide plate, or onto standard microscope slides for visual observations and cell counting. Silicon/silicon dioxide plates $\left(10 \times 10 \mathrm{~mm}^{2}\right)$ with etched silicon windows of $2 \times 2 \mathrm{~mm}^{2}$ in the center of the plate are used as sensor surfaces.

Table I Examples of spacer-link biotinylated compounds

\begin{tabular}{lll}
\hline Compound & Length of linker & Source \\
\hline aSulfo-NHS-LC-Biotin & 12 atoms & Pierce \\
'Biotin-XX-NHS & 13 atoms & Calbiochem \\
'Biotin-TEG & 15 atom3 & Pierce \\
dSulfo-NHS-LC-LC-Biotin & 19 atoms & Pierce \\
\hline
\end{tabular}

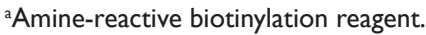

${ }^{b} \mathrm{~N}$-Hydroxysuccinimide (NHS).

'Biotin TEG: 15 atom triethylene glycol spacer.

'Amine-reactive biotinylation reagent, double long chain.

Table 2 Selected $\mathrm{K}^{+}$ion channel blockers

Tetraethylammonium 4-aminopyridine Apamin

For example,4-aminopiridine and apamin gates are to be constructed using the reaction of the Sulfo-NHS-LC-LC-Biotin (water soluble) with 4-aminopiridine and apamin, respectively, making the amide bond with the linker.

\section{Results and Discussion(Table 3 )}

\section{Advantages of the molecular switch}

i. The main advantages of the molecular switch as a transduction element in biosensors compared to many other transduction mechanisms are that they employ an amplification mechanism. The molecular switch is unlimited by the mass transport or by the bulk change effects.

ii. The molecular switch directly represents the primary analytical signal. It is a linear, sensitive and rapid chemo electric transducer. In comparison, many biosensors employing fluorescence as the transduction mechanisms are indirect sensors. They use separately labeled species, which are detected after binding, and therefore they are not very rapid. Furthermore, they require complex optical systems and sophisticated signal processing for high sensitivity.

iii. The molecular switch consumes no external power, is uniquely very small, rugged, and low cost yet is capable of detecting very low numbers of binding events.

iv. The molecular switch designed here is about 50 times smaller than a genetically engineered pore-forming protein. ${ }^{4}$ A major component of the switch is a polyene antibiotic, amphotericin $\mathrm{B}$, which makes it resistant to many bacteria. In contrast, the protein-based pores are subject to bacterial infections without proper protection.

v. The molecular switch is different from the gramicidin ionchannel. ${ }^{3}$ In the gramicidin channel, composed of two aligned antibiotic molecules, the "on" and "off" states are achieved by the displacement of one, relatively large, gramicidin molecule, while triggering the molecular switch requires only the movement of a small molecular gate. Furthermore, the modular design of the molecular switch allows the employment of different ion channels and gates and therefore offers a transduction mechanism of large dynamic range.

Table 3 Features of the Molecular Switch

\section{Features of the molecular switch}

High Sensitivity from I To I00 Binding Events

Gain I' $10^{6}$

Real time operation

Modular molecular design

Can be triggered by a large variety of sensing elements

Reversible

Electrical output signal

Small size and planar architecture

\section{Estimate of amplification of the molecular switch}

The binding of a single analyte molecule can open a molecular switch and facilitate a flux of one million ions per second. Let us consider the momentary binding of specific target molecules to antibodies on the surface of a membrane. If for example, the binding molecules are amino acids, then the time constant of the adsorption is $10^{-4}-10 \mathrm{sec}$. The highest electrical charge each of them can bring to the interface is $1.6 \times 10^{-19} \mathrm{C}$. The average charging current is $\mathrm{I}=\mathrm{dQ} /$ $\mathrm{dt}=1.6 \times 10^{-15}-1.6 \times 10^{-20} \mathrm{~A}$. If the binding of the target molecule causes the molecular switch to open an ion channel of $10-100 \mathrm{pS}$ at $100 \mathrm{mV}$, this will result in a channel current $I=10^{-12}-10^{-11} \mathrm{~A}$. In that case, the average amplification coefficient, $\mathrm{K}_{\mathrm{a}}=\mathrm{I} / \mathrm{I}$, can be estimated as $10^{6}$. The measurement of single ion channel currents is the known technology. ${ }^{2}$

\section{Application of molecular switches}

The single-molecule selectivity and specificity of the molecular recognition together with the high amplification have a promise to be utilized in many applications. ${ }^{16}$ Sensitive and molecular switches can be used for high-throughput screening of, drugs, environmental monitoring, food safety, and biowarefare control. ${ }^{17}$ Molecular 
switches can be used for detection of multiple pathogens. ${ }^{18}$ The study of molecular switches is expected to lead to the development of new sensors for a wide range of molecules.

\section{Conclusion}

We have designed a molecular switch capable of converting a single binding event into the movement of about one million ions per second. In this switch, a single binding, amplified by the release of the stored free electrochemical energy, leads to a dynamic signal that is large compared to the noise in the measuring system. The molecular switches in this work are artificial ion channels constructed by modular design from molecular pores and gates. The molecular switches can be activated by antibodies, nucleic acids, peptides, and ion sensitive molecules.

\section{Acknowledgement}

Supported by grants from DARPA, MDA972-00-1-0011 and NIST, 70 NANB14H324

\section{Conflict of interest}

The author declares no conflict of interest.

\section{References}

1. Alberts B, Bray D, Lewis J, et al. Molecular biology of the cell. 3rd ed. New York, USA: Garland Publishing; 1994;22(3):164.

2. Hille B. Ionic channels of excitable membranes. Journal of Neuroscience Research. 1984;13(4):599-600.

3. Cornell BA, Braach Maksvytis VL, King LG, et al. A biosensor that uses ion-channel switches. Nature. 1997;387(6633):580-583.

4. Braha $\mathrm{O}$, Walker B, Cheley $\mathrm{S}$, et al. Designed protein pores as components for biosensors. Cell chemical biology. 1997;4(7):497-505.

5. Sykora J, Yilma S, Neely WC, et al. Amphotericin B and cholesterol in monolayers and bilayers. Langmuir. 2003;19(3):858-864.

6. Yilma S, Cannon Sykora J, Samoylov A, et al. Large-conductance cholesterol-amphotericin B channels in reconstituted lipid bilayers. Biosensors and Bioelectron. 2007;22(7):1359-1367.
7. Fujii G, Chang JE, Coley T, et al. The formation of amphotericin B ion channels in lipid bilayers. Biochemistry. 1997;36(16):4959-4968.

8. Yilma S, Liu N, Samoylov A, et al. Amphotericin B channels in phospholipid membrane-coated nanoporous silicon surfaces: Implications for photovoltaic driving of ions across membranes. Biosensors and Bioelectron. 2007;22(8):1605-1611.

9. Yilma S, Sykora JC, Lo TT, et al. Photovoltaic driving of current through amphotericin B/cholesterol channels. Biophysical Journal. 2003;84(2 part 2):51a.

10. Silberstein A. Conformational analysis of amphotericin B - Cholesterol channel complex. J Membr Biol. 1998;162(2):117-126.

11. Samoylov AM, Samoylova TI, Hartell MG, et al. Recognition of cellspecific binding of phage display derived peptides using an acoustic wave sensor. Biomol Eng. 2002;18(6):269-272.

12. Samoylov AM, Samoylova TI, Pathirana ST, et al. Peptide biosensor for recognition of cross-species cell surface markers. J Mol Recognit. 2002;15(4):197-203.

13. Yilma S, Liu N, Samoylov A, et al. Amphotericin B channels in phospholipid membrane-coated nanoporous silicon surfaces: Implications for photovoltaic driving of ions across membranes. Biosensors Bioelectron. 2007;22(8):1605-1611.

14. Zaytseva NV, Montagna RA, Baeumner AJ. Microfluidic biosensor for the serotype-specific detection of Dengue virus RNA. Anal Chem. 2005;77(23):7520-7527.

15. Attwood SJ, Simpson AM, Stone R, et al. A simple bio conjugate attachment protocol for use in single molecule force spectroscopy experiments based on mixed self-assembled monolayers. Int $J$ Mol Sci. 2012;13(10):13521-13541.

16. Turner APF. Biosensors - Switching channels makes sense. Nature. 1997;387:555-557.

17. Steller L, Kreir M, Salzer R.et al. Natural and artificial ion channels for biosensing platforms. Anal Bioanal Chem. 2012;402(1):209-230.

18. Sang Kyu Lee, Luis G Cascão Pereira, Rafael F Sala, et al. Ion channel switch array: A biosensor for detecting multiple pathogens. Industrial Biotechnology. 2005;1(1):26-31. 